\title{
Study of Monte Carlo Simulator for Estimation of Anti-Scatter Grid Physical Characteristics on IEC 60627:2013-Based
}

\author{
Woo-Hyun Chung*, Sang-Hyun Lee \\ Research \& Development Center, JPI Healthcare Co., Ltd., Ansan-si, Republic of Korea \\ Email address: \\ chungwh@jpi.co.kr (Woo-Hyun Chung), leesh@jpi.co.kr (Sang-Hyun Lee) \\ ${ }^{*}$ Corresponding author
}

\section{To cite this article:}

Woo-Hyun Chung, Sang-Hyun Lee. Study of Monte Carlo Simulator for Estimation of Anti-Scatter Grid Physical Characteristics on IEC 60627:2013-Based. American Journal of Physics and Applications. Vol. 6, No. 2, 2018, pp. 35-42. doi: 10.11648/j.ajpa.20180602.12

Received: December 27, 2017; Accepted: January 9, 2018; Published: January 19, 2018

\begin{abstract}
The anti-scatter grid (grid) is a major component of X-ray imaging devices, improving the quality of the image by removing X-rays scattered while passing through the subject. However, problems such as image distortions or increasing the dose of a patient unnecessarily may result if the grid is not suitable for a specific digital x-ray receptor (detector.) Selecting a suitable grid can take significant work and testing due to the wide range of specifications and physical characteristic of both grid and digital imaging x-ray detectors. In order to reduce the time cost and to improve the accuracy of selecting a suitable grid, this study implemented a Monte Carlo simulation for estimating the physical characteristics of the grid and verified the accuracy of the result by comparing with the physical characteristics of the actual grid. For the verification, this study compared the estimated physical characteristics with the measured physical characteristics for ten (10) grids with different specifications. The physical characteristics were measured at RQR (Radiation Qualities in Radiation Beams emerging from the X-ray Source Assembly) 4/6/8/9 of the Radiation conditions and analyzed Transmission of Primary radiation (Tp), Transmission of Total radiation ( $\mathrm{Tt}$ ) and Transmission of Scattered radiation (Ts) of the physical characteristics of the grid. As a result of the analysis, less than $1 \%$ average deviation between simulation and physical measurement was observed with all ten (10) grids. The changes of the physical characteristics as the specifications (line density and ratio) of the grid changed were also evaluated, and found to have a Pearson's correlation coefficient of 0.998 between simulation and measurements. From the above results, the proposed program in this paper is judged reasonable as a grid physical characteristics prediction program.
\end{abstract}

Keywords: Anti-Scatter Grid, IEC 60627:2013, Grid Physical Characteristics, Monte Carlo Simulation, MCNP

\section{Introduction}

$\mathrm{X}$-ray is one of the greatest discoveries of the past century, and $\mathrm{X}$-ray scanners are used in various fields, including medical, non-destructive testing, and security. The antiscattered grid was first introduced in 1913 and was used to improve image quality by removing scattering lines created during X-ray imaging process. The scattered ray refers to the $\mathrm{X}$-ray that loses its straightness and gets refracted when passing through the subject. If this scattered ray reaches the detector, it results in an image quality degradation by making the image blurry. The grid is placed between the image receptor and the subject, improving the quality of X-ray images through removal of scattered rays and thus contributing to accuracy of diagnosis. However, using an unoptimized grid per X-ray imaging receptor may create problems such as image distortion, increased dose of x-ray for patient, etc. To prevent this, it is critical to use the grid which is optimized per X-ray imaging receptor used. The Xray imaging device and the grid have a wide range of specifications and characteristics, requiring a great deal of time and effort for the selection of optimized grid for X-ray imaging device.

This study developed simulations to predict the physical characteristics of the grid based on Monte Carlo method and verified the accuracy of the simulation by comparing to 
physical characteristics of the grid.

Chapter 2 introduces a grid used for simulation and chapter 3 describes the characteristic values for extraction. In chapter 4 describe how to extract the characteristic values and the device composition and demonstrates the performance in accordance with the experiment in chapter 5.

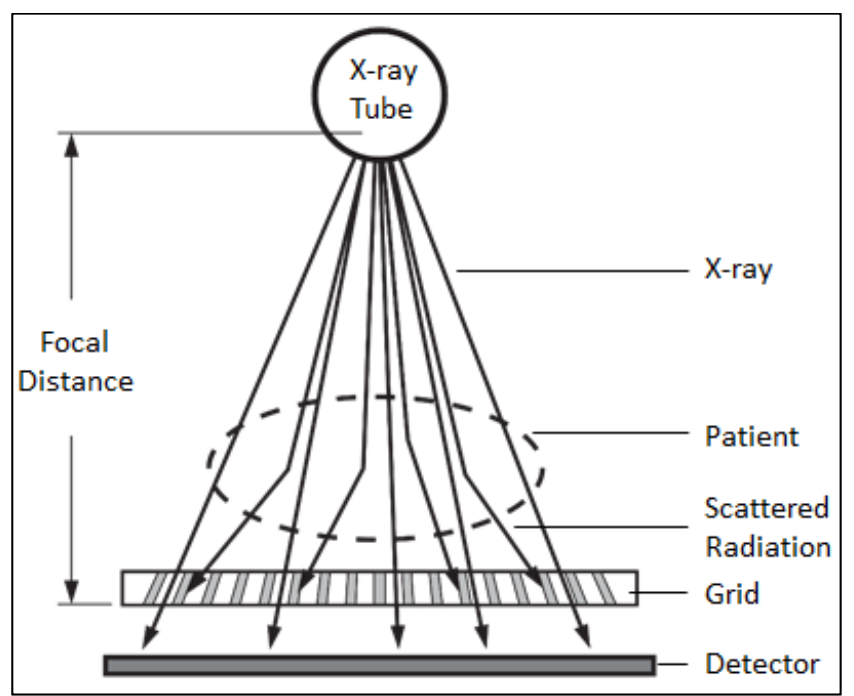

Figure 1. Acquisition of x-ray image with grid in DR system.

\section{The Composition and Specifications of Grid}

Grid consists of pairs of interspacer strip and absorber strip with width of $60 \sim 400 \mu \mathrm{m}$ and $15 \sim 50 \mu \mathrm{m}$ respectively. The lowdensity material such as aluminum, etc. is used for interspacer and it lets primary radiation pass through to reach the detector. High-density material such as lead is used for absorber and it absorbs and removes the scattered rays that are created when $\mathrm{x}$-ray passes through the subject. Line density refers to the number of pairs of inter-spacer and absorber strip in a given unit distance and Line-Pairs/CM (LP/CM) or Line-Pairs/Inch (LP/IN) is used as the unit. The line density is an important factor as it determines the occurrence of Moiré phenomenon in image and physical characteristics of grid. Grid ratio stands for the ratio of the grid height against the width of interspacer and it is also one of the main factors that determines the grid physical characteristics. In the grid, each pair of interspacer and absorber stand in the direction of the x-ray source at a specific angle based on straightness of the x-ray from the source. This angle alignment is determined with consideration to the distance from the bottom surface of grid to the x-ray source, referred to as FD (Focal Distance). Thin aluminum or carbon fiber sheets are applied to both sides of grid surfaces to protect the grids. Type of material and the thickness used for the cover also has impact on grid's physical characteristics.

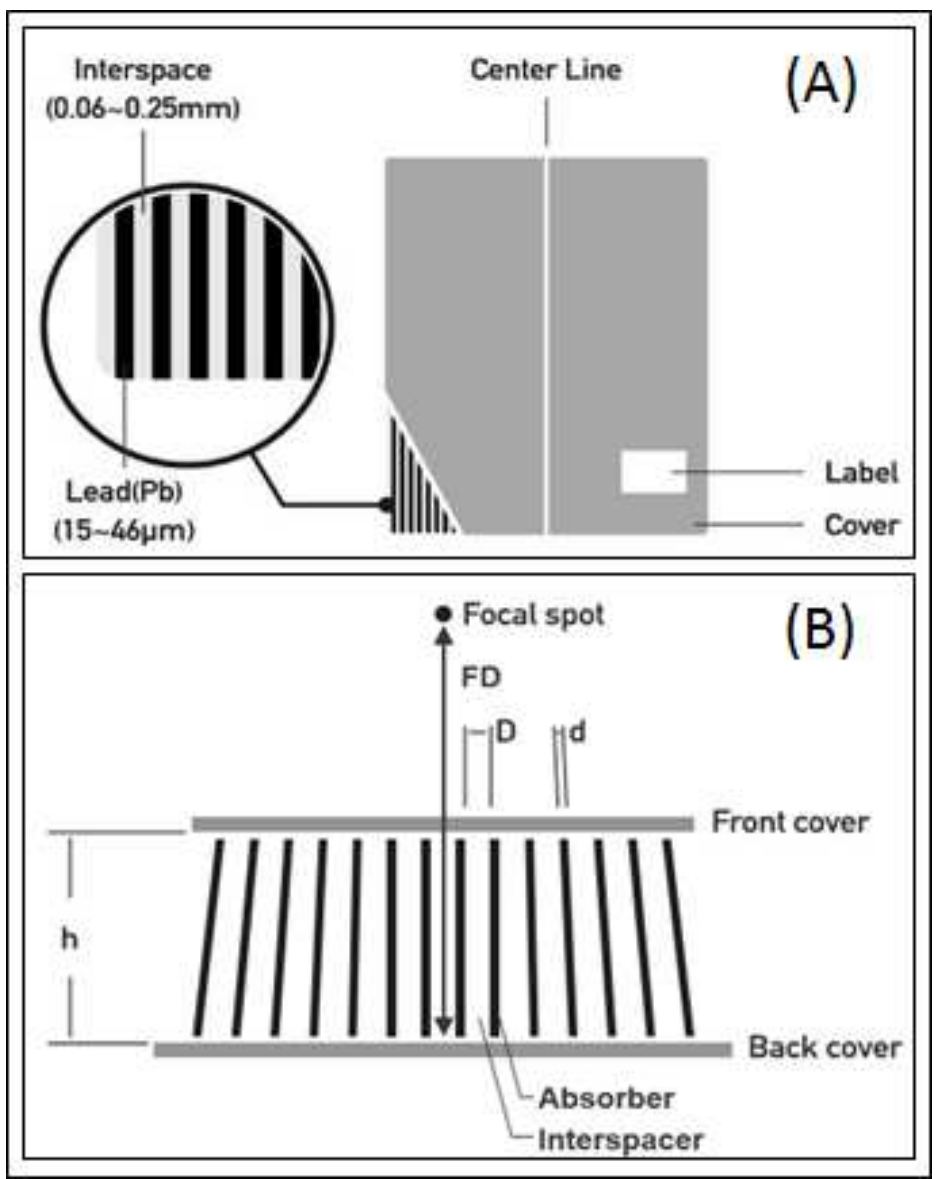

Figure 2. The structure of Grid. (A) Plane view of Grid, (B) Side view of Grid. 


\section{Physical Characteristics of Gird}

In general, There are 7 physical characteristics of the proper grid required for the X-ray imaging, and it must follow IEC (International Electrotechnical Commission) 60627: 2013 (Diagnostic X-ray imaging equipment Characteristics of general purpose and mammographic antiscatter grids) that is enacted by International Electrotechnical Commission (IEC) [6]. Three of the physical characteristics are obtained from the actual measurement, which are Tp (Transmission of Primary radiation), Tt (Transmission of Total radiation), and Ts (Transmission of Scattered radiation.) B (Grid Exposure Factor), K (Contrast Improvement Ratio), $\Sigma$ (Selectivity), and Q (Image Improvement Factor) are four physical characteristics, which are obtained by calculating measured values,

Tp refers to the amount of primary $x$-ray radiation from the $\mathrm{x}$-ray tube that actually reaches the detector after passing through grid. Higher value for Tp means better performance.

$\mathrm{Tp}$ can be calculated according to the following formula [7].

$$
T p=\frac{P_{g}}{P_{n g}}
$$

$\mathrm{P}_{\mathrm{g}}$ : Primary radiation with grid

$\mathrm{P}_{\mathrm{ng}}$ : Primary radiation without grid

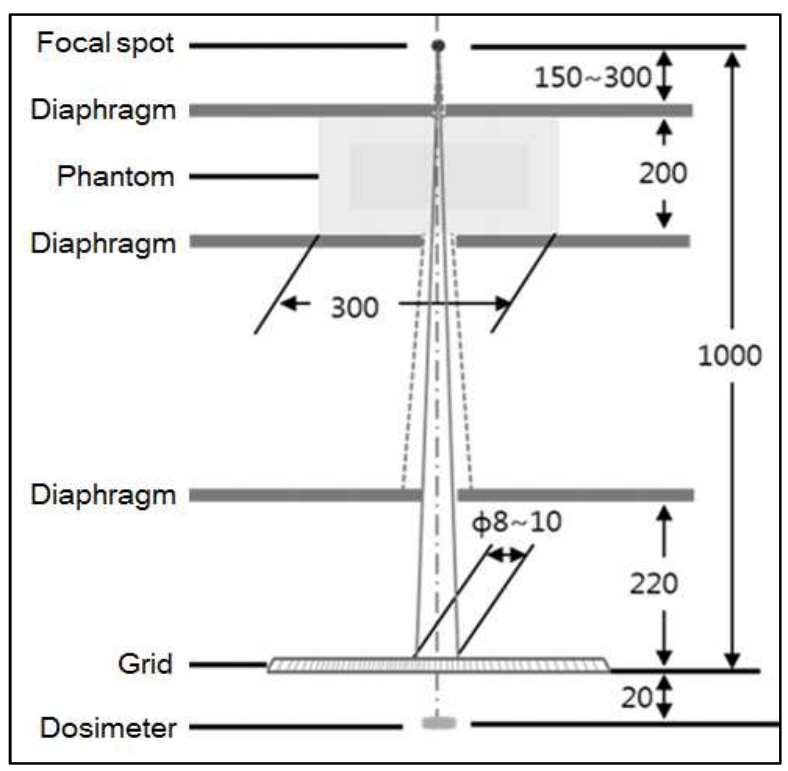

Figure 3. Measurement geometry for Transmission of Primary radiation.

Ts refers to the amount of the scattered x-ray radiation generated while the $\mathrm{x}$-ray passing through the object that actually reaches the detector even after going through the grid. Lower value for Ts means better performance. Ts can be calculated according to the following formula.

$$
T s=\frac{s_{g}}{s_{n g}}
$$

$\mathrm{S}_{\mathrm{g}}$ : Scatter radiation with grid

$\mathrm{S}_{\mathrm{ng}}$ : Scatter radiation without grid

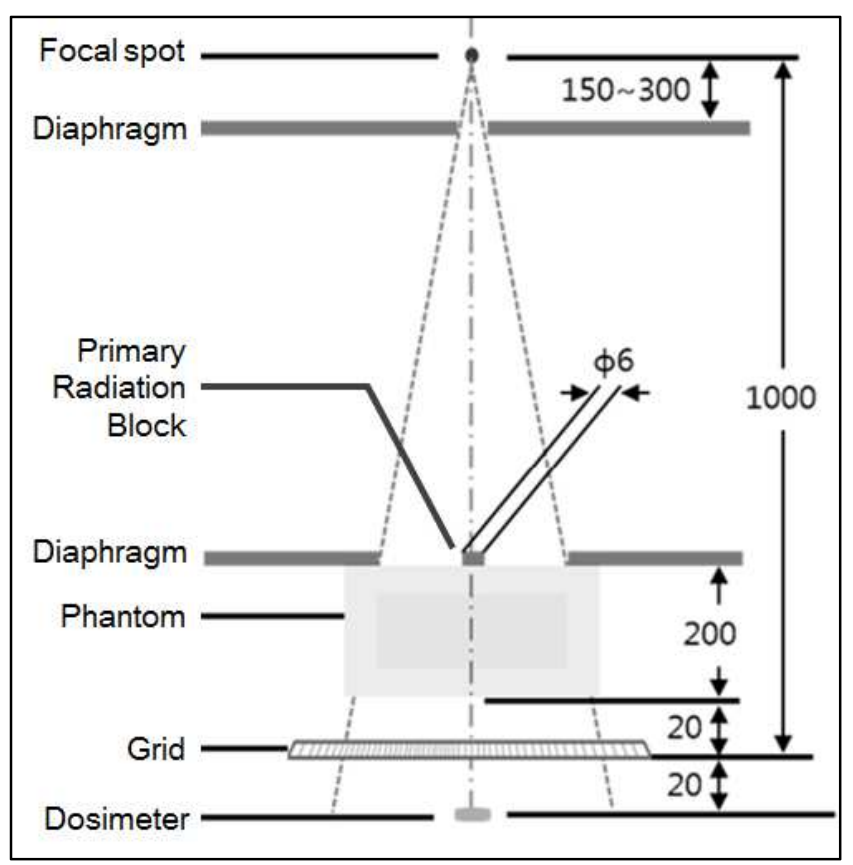

Figure 4. Measurement geometry for Transmission of Total radiation.

$\mathrm{Tt}$ refers to the amount of both primary and scattered $\mathrm{x}$ rays that reach the detector after passing through the grid. Higher value for $\mathrm{Tt}$ means better performance. $\mathrm{Tt}$ can be calculated according to the following formula.

$$
T t=\frac{T_{g}}{T_{n g}}
$$

$\mathrm{T}_{\mathrm{g}}$ : Total radiation with grid

$\mathrm{T}_{\mathrm{ng}}^{\mathrm{g}}$ : Total radiation without grid

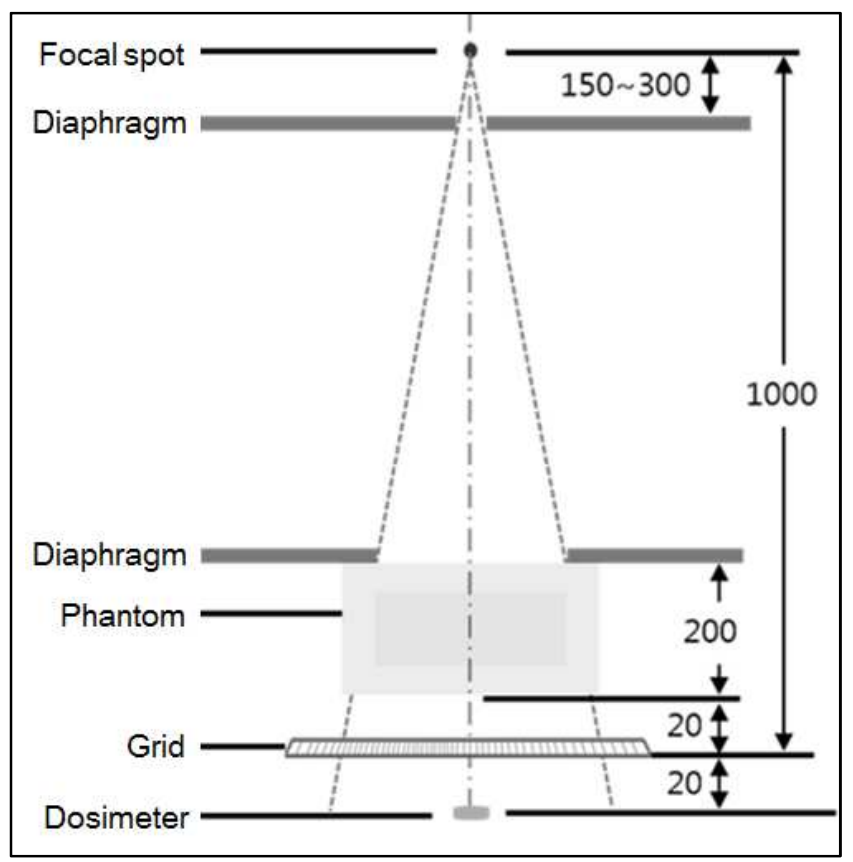

Figure 5. Geometry Measurement for Transmission of Scattered radiation. 
$\mathrm{B}$ stands for the degree indicator of the radiation dose that is increased to compensate for X-rays which is reduced by the Xray grid and the lower $\mathrm{B}$ means the better the performance. $\mathrm{B}$ can be calculated according to the following formula.

$$
B=\frac{1}{T t}
$$

$\mathrm{K}$ stands for the effect indicator of the contrast which is improved by using the X-ray grid and the higher $\mathrm{K}$ means the better the performance. $\mathrm{K}$ can be calculated according to the following formula.

$$
K=\frac{T p}{T t}
$$

$\Sigma$ stands for the proportion indicator of the transmitted primary X-ray by comparing with the scattered X-ray when using the grid and the higher $\Sigma$ means the better the performance. $\Sigma$ can be calculated according to the following formula.

$$
\Sigma=\frac{T p}{T s}
$$

Q stands for showing how much the SNR (Signal to Noise Ratio) of detector increases when using the grid and the higher Q means the better the performance. Q is calculated according to the following formula.

$$
Q=\frac{T p^{2}}{T t}
$$

\section{Materials \& Methods}

1) IEC Test Fixture

In this study, the IEC Test Fixture, in Figure 6, is used to measure three physical characteristics of grid's, Tp, Tt, and Ts. IEC Test Fixture is a device based on IEC 60627: 2013 standards. The Toshiba's E7242X X-ray tube with tube voltage range of $40 \sim 125 \mathrm{kV}$, maximum current of 290 to $800 \mathrm{~mA}$, and a focal spot of 0.6 and $1.5 \mathrm{~mm}$ is used for this test fixture. The Toreck's EY-1300 is used as radiation dosimeter and the scintillator is $\mathrm{Gd} 2 \mathrm{O} 2 \mathrm{~S}$ : Tb with an area density of $68 \mathrm{mg} / \mathrm{cm}^{2}$, and the PMT (Photomultiplier tube) is used as a photo-detector.

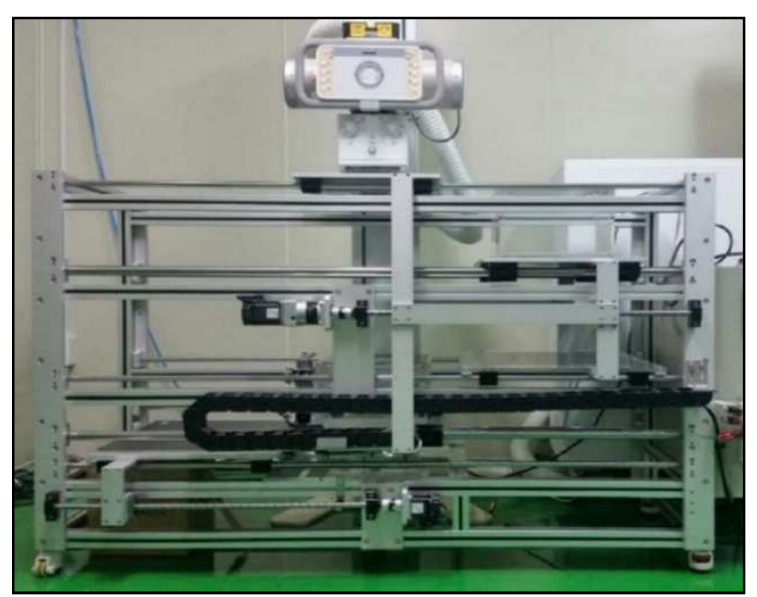

Figure 6. IEC Test Fixture.

\section{2) Grid Specification}

Table 1 shows the list of ten grids used to compare the results of simulation data against the actual physical characteristics.

Table 1. Grid specifications used for physical characteristics testing.

\begin{tabular}{lllll}
\hline No. & Line density (lp/in) & Grid ratio & FD (cm) & Cover \\
\hline 1 & 85 & $8: 1$ & 100 & $\mathrm{Al}$ \\
2 & 103 & $6: 1$ & 100 & $\mathrm{Al}$ \\
3 & 103 & $8: 1$ & 100 & $\mathrm{Al}$ \\
4 & 103 & $8: 1$ & 180 & $\mathrm{Al}$ \\
5 & 103 & $12: 1$ & 100 & $\mathrm{Al}$ \\
6 & 103 & $12: 1$ & 180 & $\mathrm{Al}$ \\
7 & 178 & $8: 1$ & 100 & $\mathrm{C}$ \\
8 & 200 & $8: 1$ & 100 & $\mathrm{Al}$ \\
9 & 200 & $12: 1$ & 100 & $\mathrm{Al}$ \\
10 & 215 & $8: 1$ & 100 & $\mathrm{C}$ \\
\hline
\end{tabular}

3) Condition of the radiation

The condition of the radiation is measured by the three conditions of RQR 4, RQR 6 and RQR 9, which are the basic requirements of IEC 60627: 2013, and the additional requirement of the customer, RQR 8 . Each condition of the radiation's filter (thickness, material) are set as shown in Table 2 based on IEC 61267: 2005 [9].

Table 2. Condition of the radiation for measuring grid physical characteristics.

\begin{tabular}{lllll}
\hline No. & $\begin{array}{l}\text { Radiation } \\
\text { condition }\end{array}$ & $\begin{array}{l}\text { Tube voltage } \\
(\mathbf{k V )}\end{array}$ & $\begin{array}{l}\text { Filter thickness } \\
(\mathbf{m m})\end{array}$ & $\begin{array}{l}\text { Filter } \\
\text { material }\end{array}$ \\
\hline 1 & RQR 4 & 60 & 1.1 & $\mathrm{Al}$ \\
2 & RQR 6 & 80 & 1.2 & $\mathrm{Al}$ \\
3 & RQR 8 & 100 & 1.6 & $\mathrm{Al}$ \\
4 & RQR 9 & 120 & 1.9 & $\mathrm{Al}$ \\
\hline
\end{tabular}

4) Simulation Software

Figure 7 shows that the user interface of the program used to simulate and estimate characteristics of the grid. The program implements grid and geometry measurement of grid for physical characteristics using MCNPX as well as the GUI of program in Visual Basic language. The program receives the specification of grid (line density, absorber, inter-spacer, ratio, FD and cover type) and condition of the radiation as inputs then displays the estimated performance on the output screen from the simulation. The frequency of simulation (nps) is set as $8 \sim 14 \times 10^{8}$ in order to increase the reliability of the estimated grid performance.

5) Measurement of grid physical characteristics

The physical characteristic of the grid is measured using the IEC Test Fixture in Figure 6. The physical characteristic is measured by measuring the radiation dose in each physical characteristics measurement structure with/without the grid, then calculated the measured radiation dose according to each formula of physical characteristics. In order to improve the reliability of the radiation dose measurement, we used the average radiation dose as representative for each grid which Max/Min value is excluded from 8 times measurement out of 10 times. 


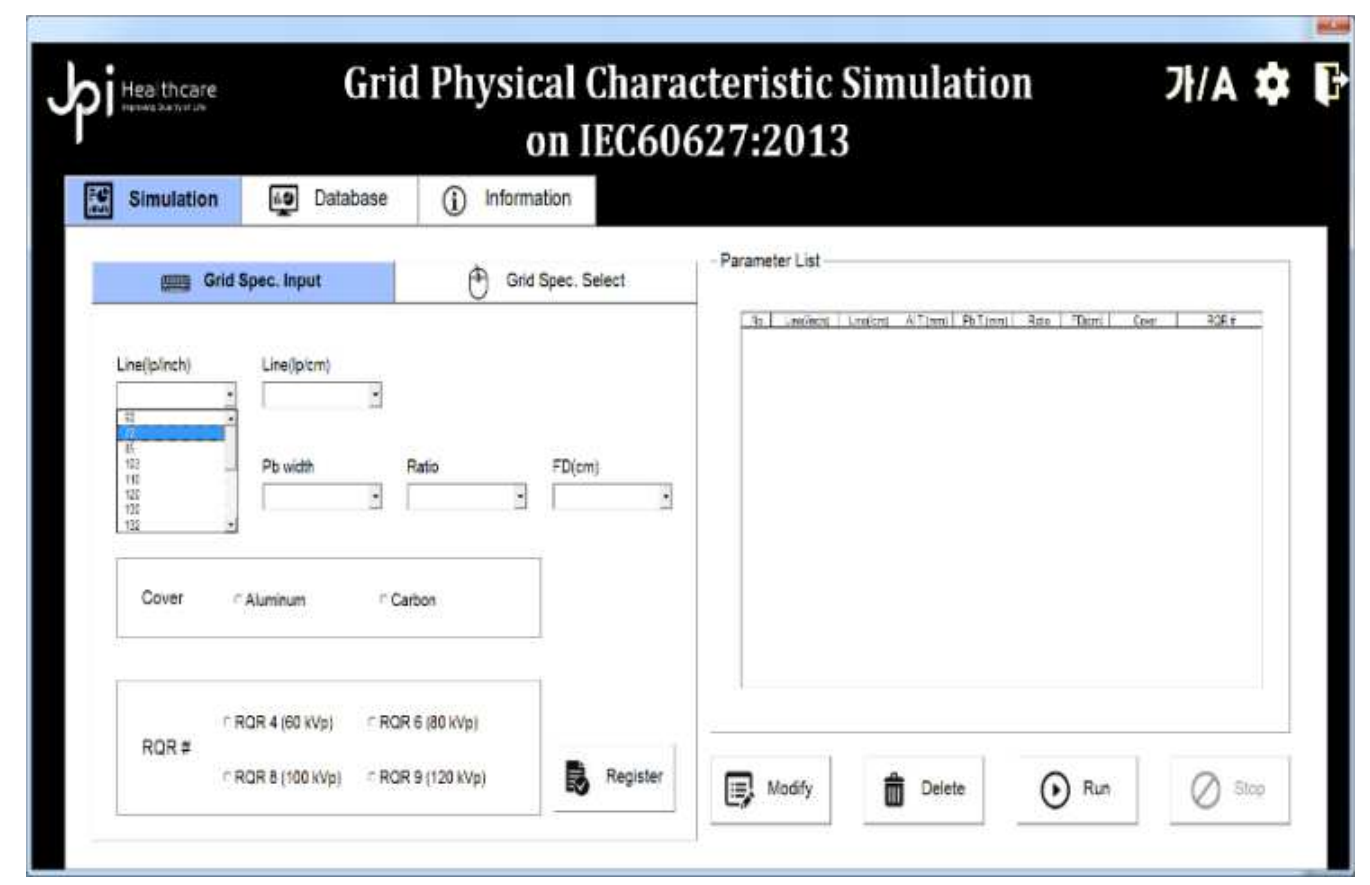

Figure 7. Program for estimation of the grid physical characteristics.

\section{Results}

1) Comparison for Characteristic of gird in RQR 4

Table 3 shows that the measured and the estimated value of physical characteristics for the grid's Tp, Tt, and Ts when the radiation condition was set to RQR 4.

Table 3. Physical characteristics of grid in RQR 4 .

\begin{tabular}{|c|c|c|c|c|c|c|c|c|c|c|}
\hline \multirow{2}{*}{ No. } & \multirow{2}{*}{ Line Density (lp/inch) } & \multirow{2}{*}{ Grid Ratio } & \multirow{2}{*}{ FD (cm) } & \multirow{2}{*}{ Cover } & \multicolumn{2}{|l|}{ Tp } & \multicolumn{2}{|l|}{$\mathbf{T t}$} & \multicolumn{2}{|l|}{ Ts } \\
\hline & & & & & Measured & Estimated & Measured & Estimated & Measured & Estimated \\
\hline 1 & 85 & $8: 1$ & 100 & $\mathrm{Al}$ & $57.89 \%$ & $58.38 \%$ & $26.00 \%$ & $25.56 \%$ & $11.25 \%$ & $10.97 \%$ \\
\hline 2 & 103 & $6: 1$ & 100 & $\mathrm{Al}$ & $64.71 \%$ & $63.46 \%$ & $32.00 \%$ & $33.02 \%$ & $18.00 \%$ & $17.05 \%$ \\
\hline 3 & 103 & $8: 1$ & 100 & $\mathrm{Al}$ & $61.11 \%$ & $61.03 \%$ & $27.66 \%$ & $27.11 \%$ & $10.00 \%$ & $11.81 \%$ \\
\hline 4 & 103 & $8: 1$ & 180 & $\mathrm{Al}$ & $61.11 \%$ & $60.47 \%$ & $26.00 \%$ & $26.18 \%$ & $9.62 \%$ & $10.77 \%$ \\
\hline 5 & 103 & 12: 1 & 100 & $\mathrm{Al}$ & $52.78 \%$ & $54.65 \%$ & $22.00 \%$ & $21.33 \%$ & $6.25 \%$ & $5.74 \%$ \\
\hline 6 & 103 & 12: 1 & 180 & $\mathrm{Al}$ & $52.78 \%$ & $54.74 \%$ & $22.00 \%$ & $21.02 \%$ & $6.10 \%$ & $5.33 \%$ \\
\hline 7 & 178 & $8: 1$ & 100 & $\mathrm{C}$ & $61.11 \%$ & $59.19 \%$ & $26.00 \%$ & $27.99 \%$ & $13.75 \%$ & $12.91 \%$ \\
\hline 8 & 200 & $8: 1$ & 100 & $\mathrm{Al}$ & $63.64 \%$ & $64.29 \%$ & $32.00 \%$ & $31.70 \%$ & $16.25 \%$ & $14.67 \%$ \\
\hline 9 & 200 & 12: 1 & 100 & $\mathrm{Al}$ & $61.11 \%$ & $61.56 \%$ & $27.66 \%$ & $26.06 \%$ & $10.00 \%$ & $9.55 \%$ \\
\hline 10 & 215 & $8: 1$ & 100 & $\mathrm{C}$ & $61.11 \%$ & $62.17 \%$ & $32.00 \%$ & $31.40 \%$ & $16.25 \%$ & $16.25 \%$ \\
\hline
\end{tabular}

In RQR 4, the mean error between the measured and the estimated value of $\mathrm{Tp}$ was $1.04 \%$ while the maximum error was $1.96 \%$. The mean error between the measured and the estimated value of $\mathrm{Tt}$ was $0.83 \%$ while the maximum error was $1.99 \%$. The mean error between the measured and the estimated value of $\mathrm{Tt}$ was $0.83 \%$ while the maximum error was $1.81 \%$. In RQR 4 , the mean error between the measured and the estimated value of all grids was $0.90 \%$ while the maximum error was $1.99 \%$.

2) Comparison for Characteristic of grid in RQR 6

Table 4 shows that the measured and the estimated value of physical characteristic for the grid's Tp, Tt, and Ts when the condition of radiation was set to RQR 6 . In RQR 6, the mean error between the measured and the estimated value of Tp was $0.81 \%$ while the maximum error was $1.80 \%$. The mean error between the measured and the estimated value of Tt was $0.86 \%$ while the maximum error was $1.96 \%$. The mean error between the measured and the estimated value of Tt was $0.88 \%$ while the maximum error was $1.82 \%$. In RQR 6 , the mean error between the measured and the estimated value of all grids was $0.85 \%$ while the maximum error was $1.96 \%$. 
Table 4. Physical characteristics of grid in RQR 6.

\begin{tabular}{|c|c|c|c|c|c|c|c|c|c|c|}
\hline \multirow{2}{*}{ No. } & \multirow{2}{*}{ Line Density (lp/inch) } & \multirow{2}{*}{ Grid Ratio } & \multirow{2}{*}{ FD (cm) } & \multirow{2}{*}{ Cover } & \multicolumn{2}{|l|}{$\mathbf{T p}$} & \multicolumn{2}{|l|}{ Tt } & \multicolumn{2}{|l|}{ Ts } \\
\hline & & & & & Measured & Estimated & Measured & Estimated & Measured & Estimated \\
\hline 1 & 85 & $8: 1$ & 100 & $\mathrm{Al}$ & $66.19 \%$ & $67.94 \%$ & $33.83 \%$ & $33.54 \%$ & $18.97 \%$ & $17.97 \%$ \\
\hline 2 & 103 & $6: 1$ & 100 & $\mathrm{Al}$ & $71.32 \%$ & $69.52 \%$ & $41.59 \%$ & $41.40 \%$ & $25.21 \%$ & $26.89 \%$ \\
\hline 3 & 103 & $8: 1$ & 100 & $\mathrm{Al}$ & $68.42 \%$ & $67.18 \%$ & $34.23 \%$ & $34.21 \%$ & $18.97 \%$ & $19.10 \%$ \\
\hline 4 & 103 & $8: 1$ & 180 & $\mathrm{Al}$ & $67.91 \%$ & $67.56 \%$ & $34.51 \%$ & $33.39 \%$ & $18.49 \%$ & $19.21 \%$ \\
\hline 5 & 103 & 12: 1 & 100 & $\mathrm{Al}$ & $62.71 \%$ & $61.92 \%$ & $26.55 \%$ & $27.16 \%$ & $9.24 \%$ & $11.06 \%$ \\
\hline 6 & 103 & 12: 1 & 180 & $\mathrm{Al}$ & $61.87 \%$ & $62.85 \%$ & $26.55 \%$ & $25.86 \%$ & $9.48 \%$ & $10.28 \%$ \\
\hline 7 & 178 & $8: 1$ & 100 & $\mathrm{C}$ & $63.31 \%$ & $63.56 \%$ & $33.63 \%$ & $35.47 \%$ & $21.01 \%$ & $21.75 \%$ \\
\hline 8 & 200 & $8: 1$ & 100 & $\mathrm{Al}$ & $71.94 \%$ & $72.40 \%$ & $41.59 \%$ & $40.88 \%$ & $27.73 \%$ & $27.73 \%$ \\
\hline 9 & 200 & 12: 1 & 100 & $\mathrm{Al}$ & $67.63 \%$ & $67.14 \%$ & $34.23 \%$ & $33.07 \%$ & $18.97 \%$ & $17.97 \%$ \\
\hline 10 & 215 & $8: 1$ & 100 & C & $69.06 \%$ & $69.05 \%$ & $41.59 \%$ & $39.63 \%$ & $27.73 \%$ & $26.87 \%$ \\
\hline
\end{tabular}

Table 5. Physical characteristics of grid in RQR 8 .

\begin{tabular}{|c|c|c|c|c|c|c|c|c|c|c|}
\hline \multirow{2}{*}{ No. } & \multirow{2}{*}{ Line Density (Ip/inch) } & \multirow{2}{*}{ Grid Ratio } & \multirow{2}{*}{ FD (cm) } & \multirow{2}{*}{ Cover } & \multicolumn{2}{|l|}{ Tp } & \multicolumn{2}{|l|}{ Tt } & \multicolumn{2}{|l|}{ Ts } \\
\hline & & & & & Measured & Estimated & Measured & Estimated & Measured & Estimated \\
\hline 1 & 85 & $8: 1$ & 100 & $\mathrm{Al}$ & $69.88 \%$ & $71.27 \%$ & $37.23 \%$ & $38.51 \%$ & $22.15 \%$ & $22.73 \%$ \\
\hline 2 & 103 & $6: 1$ & 100 & $\mathrm{Al}$ & $73.49 \%$ & $73.79 \%$ & $43.75 \%$ & $45.69 \%$ & $30.92 \%$ & $30.72 \%$ \\
\hline 3 & 103 & $8: 1$ & 100 & $\mathrm{Al}$ & $70.19 \%$ & $71.53 \%$ & $37.41 \%$ & $38.79 \%$ & $23.81 \%$ & $22.50 \%$ \\
\hline 4 & 103 & $8: 1$ & 180 & $\mathrm{Al}$ & $70.12 \%$ & $72.06 \%$ & $39.01 \%$ & $37.98 \%$ & $24.00 \%$ & $23.99 \%$ \\
\hline 5 & 103 & 12: 1 & 100 & $\mathrm{Al}$ & $65.68 \%$ & $66.72 \%$ & $29.50 \%$ & $28.96 \%$ & $14.29 \%$ & $13.12 \%$ \\
\hline 6 & 103 & 12: 1 & 180 & $\mathrm{Al}$ & $65.68 \%$ & $67.63 \%$ & $29.50 \%$ & $29.01 \%$ & $14.29 \%$ & $12.42 \%$ \\
\hline 7 & 178 & $8: 1$ & 100 & $\mathrm{C}$ & $66.07 \%$ & $64.72 \%$ & $39.01 \%$ & $39.55 \%$ & $25.33 \%$ & $26.91 \%$ \\
\hline 8 & 200 & $8: 1$ & 100 & $\mathrm{Al}$ & $73.96 \%$ & $73.09 \%$ & $45.83 \%$ & $47.59 \%$ & $34.07 \%$ & $34.59 \%$ \\
\hline 9 & 200 & 12: 1 & 100 & $\mathrm{Al}$ & $70.41 \%$ & $69.75 \%$ & $39.57 \%$ & $37.81 \%$ & $24.49 \%$ & $23.47 \%$ \\
\hline 10 & 215 & $8: 1$ & 100 & $\mathrm{C}$ & $70.83 \%$ & $69.17 \%$ & $46.48 \%$ & $45.36 \%$ & $34.67 \%$ & $33.37 \%$ \\
\hline
\end{tabular}

3) Comparison for Characteristic of grid in RQR 8

Table 5 shows that the measured and the estimated value of physical characteristics for the grid's Tp, Tt, and Ts when the condition of radiation was set to RQR 8. In RQR 8, the mean error between the measured and the estimated value of Tp was $1.25 \%$ while the maximum error was $1.95 \%$. The mean error between the measured and the estimated value of Tt was $1.18 \%$ while the maximum error was $1.94 \%$. The mean error between the measured and the estimated value of $\mathrm{Tt}$ was $0.96 \%$ while the maximum error was $1.87 \%$. In RQR 8 , the mean error between the measured and the estimated value of all grids was $1.13 \%$ while the maximum error was $1.95 \%$.

Table 6. Physical characteristics of grid in RQR 9.

\begin{tabular}{|c|c|c|c|c|c|c|c|c|c|c|}
\hline \multirow{2}{*}{ No. } & \multirow{2}{*}{ Line Density (lp/inch) } & \multirow{2}{*}{ Grid Ratio } & \multirow{2}{*}{ FD (cm) } & \multirow{2}{*}{ Cover } & \multicolumn{2}{|l|}{ Tp } & \multicolumn{2}{|l|}{$\mathrm{Tt}$} & \multicolumn{2}{|l|}{ Ts } \\
\hline & & & & & Measured & Estimated & Measured & Estimated & Measured & Estimated \\
\hline 1 & 85 & $8: 1$ & 100 & $\mathrm{Al}$ & $70.00 \%$ & $71.76 \%$ & $39.37 \%$ & $38.70 \%$ & $24.26 \%$ & $22.85 \%$ \\
\hline 2 & 103 & $6: 1$ & 100 & $\mathrm{Al}$ & $73.79 \%$ & $72.04 \%$ & $46.92 \%$ & $47.15 \%$ & $33.81 \%$ & $34.03 \%$ \\
\hline 3 & 103 & $8: 1$ & 100 & $\mathrm{Al}$ & $71.53 \%$ & $69.85 \%$ & $40.42 \%$ & $38.77 \%$ & $26.09 \%$ & $24.14 \%$ \\
\hline 4 & 103 & $8: 1$ & 180 & $\mathrm{Al}$ & $70.34 \%$ & $70.25 \%$ & $40.00 \%$ & $39.17 \%$ & $25.90 \%$ & $26.20 \%$ \\
\hline 5 & 103 & 12: 1 & 100 & $\mathrm{Al}$ & $66.67 \%$ & $66.09 \%$ & $31.50 \%$ & $31.50 \%$ & $16.18 \%$ & $15.43 \%$ \\
\hline 6 & 103 & 12: 1 & 180 & $\mathrm{Al}$ & $66.67 \%$ & $66.30 \%$ & $31.50 \%$ & $30.39 \%$ & $16.18 \%$ & $15.34 \%$ \\
\hline 7 & 178 & $8: 1$ & 100 & $\mathrm{C}$ & $65.79 \%$ & $66.06 \%$ & $40.31 \%$ & $42.10 \%$ & $27.34 \%$ & $29.30 \%$ \\
\hline 8 & 200 & $8: 1$ & 100 & $\mathrm{Al}$ & $73.67 \%$ & $73.18 \%$ & $48.46 \%$ & $49.97 \%$ & $37.41 \%$ & $37.18 \%$ \\
\hline 9 & 200 & 12: 1 & 100 & $\mathrm{Al}$ & $72.00 \%$ & $70.04 \%$ & $40.94 \%$ & $39.27 \%$ & $26.47 \%$ & $25.91 \%$ \\
\hline 10 & 215 & $8: 1$ & 100 & $\mathrm{C}$ & $72.00 \%$ & $70.25 \%$ & $48.46 \%$ & $47.50 \%$ & $37.41 \%$ & $35.60 \%$ \\
\hline
\end{tabular}

4) Comparison for Characteristic of grid in RQR 9

Table 6 shows that the measured and the estimated value of physical characteristics for the grid's Tp, Tt, and Ts when the radiation condition was set to RQR 9. In RQR 9, the mean error between the measured and the estimated value of Tp was $1.07 \%$ while the maximum erro was $1.96 \%$. The mean error between the measured and the estimated value of
Tt was $1.04 \%$ while the maximum error was $1.94 \%$. The mean error between the measured and the estimated value of Tt was $0.96 \%$ while the maximum error was $1.87 \%$. In RQR 8 , the mean error between the measured and the estimated value of all grids was $1.13 \%$, and the maximum error was $1.95 \%$. 

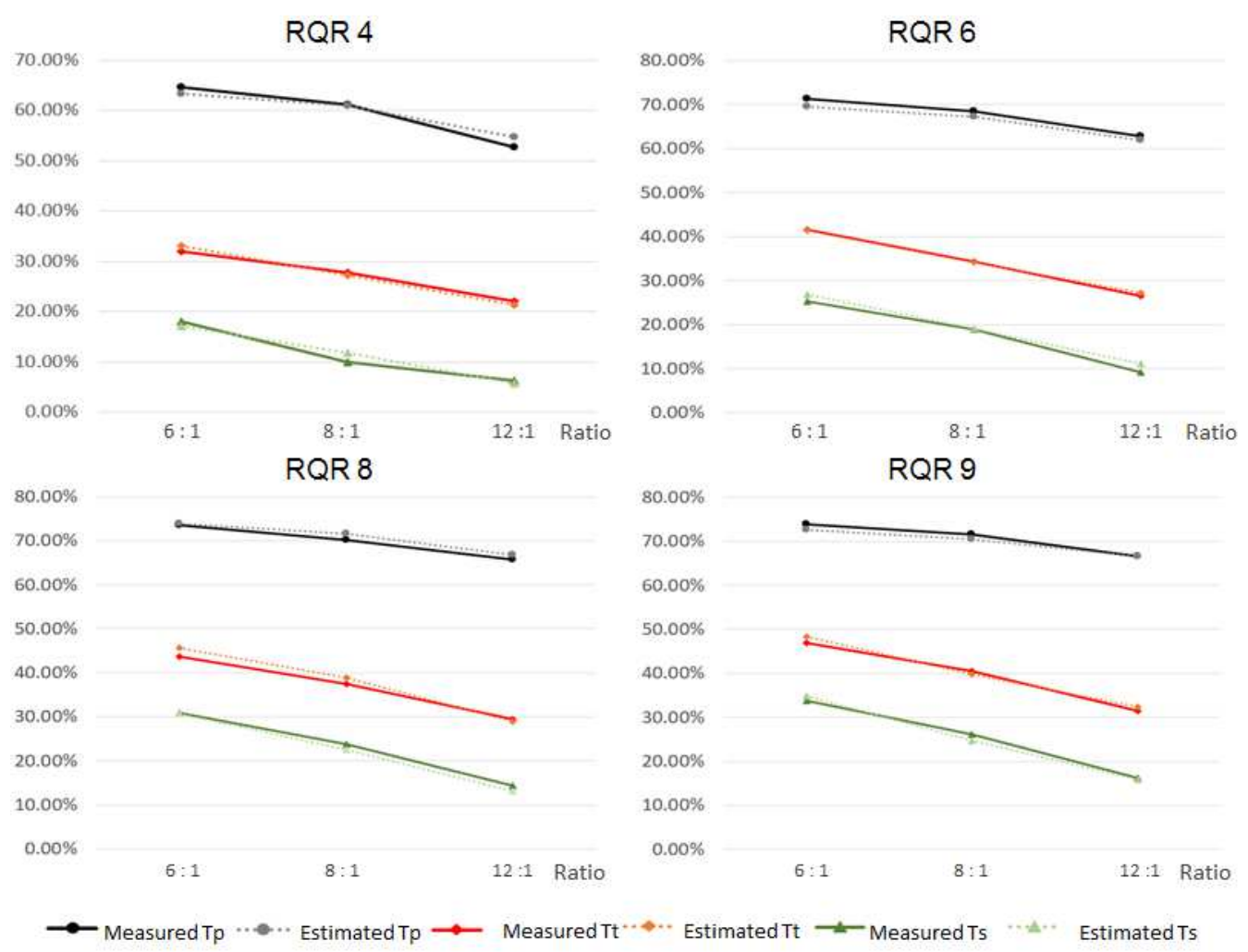

Figure 8. Change in Grid Performance per Grid Ratio.

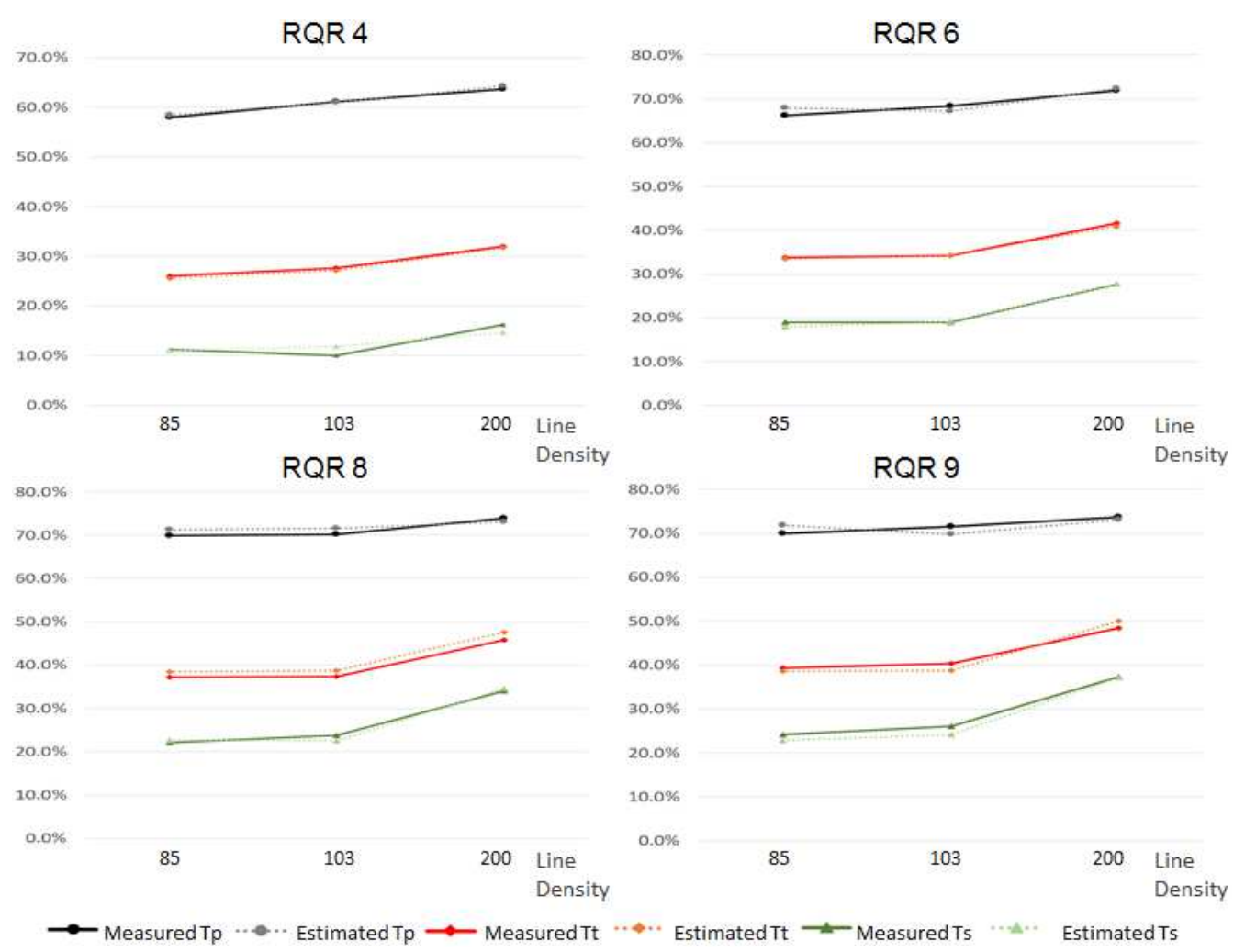

Figure 9. Change in Grid Performance Per Line Density.

5) Changes of characteristics according to the ratio

Figure 8 shows a graph of the change in physical characteristics according to grid ratio and the grid has same specification (Line Density 103 LP/in, FD 100 cm, Al cover) except grid ratio. In RQR 6, the measured and the estimated value of $\mathrm{Tp}$ are decreased by $8.6 \%$ and $7.6 \%$, respectively as the ratio increases from $6: 1$ to $12: 1$. In the same condition, the measured and the estimated value of $\mathrm{Tt}$ are decreased by 
$15.0 \%$ and $14.2 \%$, respectively. Also, the measured and the estimated value of Ts are decreased by $16.0 \%$ and $15.8 \%$, respectively.

In RQR 8 , the measured and the estimated value of Tp are decreased by $7.8 \%$ and $7.1 \%$, respectively as the ratio increases from $6: 1$ to $12: 1$. In the same condition, the measured and the estimated value of $\mathrm{Tt}$ are decreased by $14.3 \%$ and $16.7 \%$, respectively. Also, the measured and the estimated value of Ts are decreased by $16.6 \%$ and $17.6 \%$, respectively.

As the grid ratio increases, the physical characteristic Tp, $\mathrm{Tt}$, and Ts of measured and estimated physical characteristic was shown as Pearson correlation coefficient 0.998. [10]. Therefore, it can be considered as measured and estimated physical characteristics show a similar tendency of change.

6) Changes of characteristics according to the line density

Figure 9 shows a graph of the change in physical characteristics according to the line density and the grid has same specification (Grid Ratio 8:1, FD $100 \mathrm{~cm}$, Al cover) except line density. In RQR 6, the measured and the estimated value of $\mathrm{Tp}$ are increased by $5.8 \%$ and $4.5 \%$, respectively as the ratio increases from $6: 1$ to $12: 1$. In the same condition, the measured and the estimated value of Tt are increased by $7.8 \%$ and $7.3 \%$, respectively. Also, the measured and the estimated value of Ts are increased by $8.8 \%$ and $9.8 \%$, respectively.

In RQR 8, the measured and the estimated value of Tp are increased by $4.1 \%$ and $1.8 \%$, respectively as the ratio increases from $6: 1$ to $12: 1$. In the same condition, the measured and the estimated value of $\mathrm{Tt}$ are decreased by $8.6 \%$ and $9.1 \%$, respectively. Also, the measured and the estimated value of Ts are decreased by $11.9 \%$ and $11.9 \%$, respectively.

As the line density changes, the physical characteristic Tp, $\mathrm{Tt}$, and Ts of measured and estimated physical characteristic was shown as Pearson correlation coefficient 0.998 . Therefore, it can be considered as measured and estimated physical characteristics show a similar tendency of change.

Table 7. Difference of actual and estimated grid physical characteristics.

\begin{tabular}{llllll}
\hline \multirow{2}{*}{$\begin{array}{c}\text { No. } \\
\text { Radiation } \\
\text { condition }\end{array}$} & \multicolumn{4}{l}{$\begin{array}{l}\text { Average error rate between the measured and } \\
\text { estimated values }\end{array}$} \\
\cline { 3 - 6 } & & Tt & Tp & Ts & Average \\
\hline 1 & RQR 4 & $0.83 \%$ & $1.04 \%$ & $0.83 \%$ & $0.90 \%$ \\
2 & RQR 6 & $0.86 \%$ & $0.81 \%$ & $0.88 \%$ & $0.85 \%$ \\
3 & RQR 8 & $1.18 \%$ & $1.25 \%$ & $0.96 \%$ & $1.13 \%$ \\
4 & RQR 9 & $1.04 \%$ & $1.07 \%$ & $1.00 \%$ & $1.04 \%$ \\
\multicolumn{2}{l}{ Average } & $0.98 \%$ & $1.04 \%$ & $0.92 \%$ & $0.98 \%$ \\
\hline
\end{tabular}

\section{Conclusion}

This study developed a program for estimating a grid's physical characteristics based on IEC 60627: 2013, and compared the physical characteristics of measured grids with the estimates. The difference between the measured and the estimated physical characteristics averaged $0.90 \%$ (maximum $1.99 \%$ ) in RQR 4, 0.85\% (maximum 1.96\%) in RQR 6, 1.13\% (maximum 1.95\%) in RQR 8 and $1.04 \%$ (maximum 1.96\%) in RQR 9. The average difference in all conditions was $0.98 \%$, less than $1 \%$. Also, changes in the grid ratio and line density produced very similar variation in the measured and simulated physical characteristics of the grids.

\section{References}

[1] W. C. Röntgen., et al. "Über eine neue Art Von Strahlen" Würzburger Berichte (1895), pp. 132-141.

[2] Bucky G, "A Grating-Diaphragm to Cut off Secondary Rays from the Object" Archives of the Roentgen Ray 18 (1913): 6-9.

[3] Hondius Boldingh, Willem., et al. "Grids to reduce scattered X-rays in medical radiography" Philips Research Reports Supplement 1 (1964).

[4] Tang, C-M., et al. "Anti-scattering X-ray grid" Microsystem technologies 4.4 (1998): 187-192.

[5] Hendee, William R., et al. "Radiography" Medical Imaging Physics, Fourth Edition (2002): 217-234.

[6] "IEC 60627:2013, - Diagnostic X-ray imaging equipment Characteristics of general purpose and mammographic antiscatter grids" International Electrotechnical Commission, 2013.

[7] Dogan Bor., et al. "Investigation of grid performance using simple image quality tests" Journal of Medical Physics, Vol. 41 (1), pp 21-28, 2016.

[8] “JPI’s Grid Book (2015) Version VIII" - www.jpi.co.kr.

[9] "IEC62220-1:2003, Medical electrical equipment Characteristics of digital X-ray imaging devices - Part 1: Determination of the detective quantum efficiency" International Electrotechnical Commission, 2003.

[10] Kent State University Libraries. "Pearson Correlation" Accessed December 05, 2017. https://libguides.library.kent.edu/SPSS/PearsonCorr. 